\title{
An Image Recognition Method for Moving Stick and Its Application
}

\author{
Mengxiang $\mathrm{Xu}^{\mathrm{a}}$, Guangyu $\mathrm{Liu}^{\mathrm{b}}$ \\ School of Automation, Hangzhou Dianzi University, Hangzhou 310018, China \\ a1203433230@qq.com, b.liu@hdu.edu.cn
}

Keywords:motion detection; Hough transform; stick object recognition; canny operator.

\begin{abstract}
The requirement and the status of the detection of the moving stick are introduced, and the Hough transform and the canny gradient are introduced to identify the movement of the object in the scene. By extracting the Canny gradient of the moving object, the Hough line detection is performed on the gradient image, and then the object is identified as a stick object. It can detect and alarm the stick like object in simple scene, so as to make sure the risk level of the sudden incident before we get to field. Finally, the feasibility of the algorithm is verified by simulation.
\end{abstract}

\section{Introduction}

With the need of security grows day by day, security and protection business has become demands of the time. Also with the development of society, as long as the social crime and unstable factors do not decrease, security and protection business will long exists and develops. As it turns out, social crime rate does not decrease with the social development and economic prosperity. Without high-tech security and protection system, the social crime rate of European, America and other developed countries would be several times, even tens of times higher than that of now.

Modern society have increasingly requirement of security, the fight happens quite often, how to prevent and detect of this kind of behavior as early as possible has great significance. For early warning signs of danger on the market at present mainly two main ways to realize, one is through sensors, the other is through image processing. Sensors can be detective and warning to light, smell, and smoke situations. Image processing can analysis and has early warning to visible behavior and their existence. At present, the image processing can carry on good monitoring on such behavior as leave over object, stolen objects and regional invasion. But in fighting, the dangerous objects are rarely analyzed. This paper puts forward the detection method of the rod shaped object's movement; it can effectively detect the behavior with a stick in the environment. It has vital significance in early detection and warning to fighting.

\section{Detection of moving objects}

Frame difference and background difference are two basic methods of motion detection. The frame difference method is to select the adjacent frames, and the time difference between the pixels and the threshold is used to extract the moving region. The subtraction of two adjacent frame image pixels would be compared with the threshold. If the difference is greater than the threshold, it is considered to be the foreground image; otherwise it is considered as a background image. We can determine the location of the target in the image by using the labeled pixel area. The basic formula is as follows:

$$
\begin{array}{ll}
|\mathrm{i}(\mathrm{t})-\mathrm{i}(\mathrm{t}-1)|<T & \text { Background }(1) \\
|\mathrm{i}(\mathrm{t})-\mathrm{i}(\mathrm{t}-1)| \geq \mathrm{T} & \text { Foreground }
\end{array}
$$

The basic principle of the background difference method is: first select an image as the background frame, and then compare the current frame with background frame, get the difference and set the threshold $\mathrm{T}$. If the difference is bigger than $\mathrm{T}$, the image is a moving target; otherwise it is considered as the background.

As is shown in Fig.1, there are two images of pedestrian detected respectively by frame difference and background difference. These two methods have their advantages and disadvantages. The advantages of the frame difference method are: high real-time, fast update speed, small amount of 
computation. Drawback is that the impact of choosing the threshold is very big. If the threshold chosen is too small, the background might be wrongly detected as moving object; if the threshold is too large, the moving object might not be detected. The background difference method can accurately segment the moving target. But background difference method also has shortcomings, such as: it is very sensitive to the change of background environment, and the selection of background image is also very important. We want to extract the whole outline of the stick like object, so we choose the background difference method.

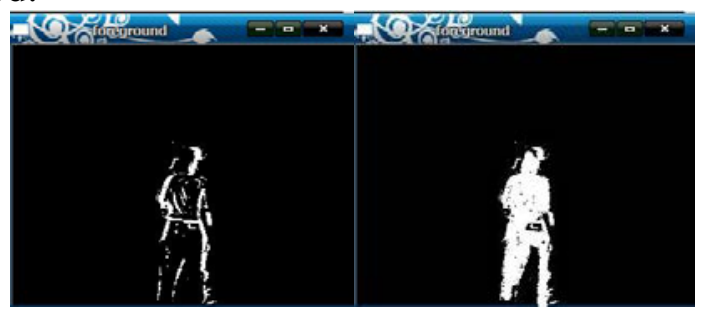

(a) Frame difference

(b) Background difference

Fig.1 Pedestrian detection

To use background difference method, we have to solve two problems: (1) the background image acquisition: background image acquisition should be at a complete still circumstance, without any movement of the object, and in some practical situations to get a pair of completely stationary background image is a little hard to accomplish; (2)the background image update: background subtraction method is only applicable to indoor environment conditions such as the ideal situation, and in the long time monitoring the background cannot be static, so it is necessary to update the background image in a period of time, to adapt to the environment changes.

The mixed Gauss background model used here is a good method. It is based on the background of the statistical method of pixel samples ${ }^{[1]}$. In a long time, the probability distribution of the sample value of pixels is used to represent the background, and then the target pixels are judged by the statistical difference. In this model, the color information of pixels is not related to each pixel.

For the Gauss distribution model of multiple peaks, the pixel points in the image are carried out according to different weights, and then the Gauss distribution is modeled by superposition. And each kind of Gauss distribution corresponds to a possible state of the color of the pixels, the Gauss distribution weights and parameters update with time ${ }^{[2]}$. In the processing of colored images, the R, G, $\mathrm{B}$ channels of pixels are independent and the variances are the same. Take random variable $\mathrm{X}$ as the observation data $\operatorname{set}\left\{X_{1}, X_{2}, \ldots, X_{n}\right\}, X_{t}=\left(R_{t}, G_{t}, B_{t}\right)$ is a pixel sample for the moment $t$. Then the sampling points are subject to the probability density function of the mixed Gauss distribution:

$$
\left\{\begin{array}{c}
\mathrm{p}\left(\mathrm{x}_{\mathrm{t}}\right)=\sum_{\mathrm{i}=1}^{\mathrm{k}} \omega_{\mathrm{i}, \mathrm{t}} \times \eta\left(\mathrm{x}_{\mathrm{t}}, \mu_{\mathrm{i}, \mathrm{t}}, \tau_{\mathrm{i}, \mathrm{t}}\right) \\
\eta\left(\mathrm{x}_{\mathrm{t}}, \mu_{\mathrm{i}, \mathrm{t}}, \tau_{\mathrm{i}, \mathrm{t}}\right)=\frac{1}{\tau_{\mathrm{i}, \mathrm{t}}^{1 / 2}} \mathrm{e}^{-\frac{1}{2}\left(\mathrm{x}_{\mathrm{t}}-\mu_{\mathrm{i}, \mathrm{t}}\right)^{\mathrm{T}} \tau_{\mathrm{i}, \mathrm{t}}{ }^{-1}\left(\mathrm{x}_{\mathrm{t}}-\mu_{\mathrm{i}, \mathrm{t}}\right)} \\
\tau_{\mathrm{i}, \mathrm{t}}=\delta_{\mathrm{i}, \mathrm{t}}{ }^{2} \mathrm{I}
\end{array}\right.
$$

Where $\mathrm{k}$ is the total number of models in the distribution model, $\eta\left(\mathrm{x}_{\mathrm{t}}, \mu_{\mathrm{i}, \mathrm{t}}, \tau_{\mathrm{i}, \mathrm{t}}\right)$ is the number $\mathrm{i}$ Gauss distribution of moment $\mathrm{t}, \mu_{\mathrm{i}, \mathrm{t}}$ is mean, $\tau_{\mathrm{i}, \mathrm{t}}$ is covariance matrix of the model, $\delta_{i, \mathrm{t}}$ is variance, $\mathrm{I}$ is a three-dimensional unit matrix, $\omega_{i, t}$ is the weights of the distribution of the Gauss of moment $t$.

The basic contents and steps of the Gauss background model are as follows:

1)The new pixel values of Xt and the current $\mathrm{K}$ models are calculated according to the following formula, and the mean deviation of the distribution model is less than or equal to $2.5 \sigma$ :

$$
\left|\mathrm{X}_{\mathrm{t}}-\mu_{\mathrm{i}, \mathrm{t}-1}\right| \leq 2.5 \sigma_{\mathrm{i}, \mathrm{t}-1}
$$

2) If the matching pattern is in line with the requirements of the background, and then the pixel is the background pixel.

3)Update the weights according to the following formula, where $\alpha$ is the learning rate. If the pattern matches, $\mathrm{M}_{\mathrm{k}, \mathrm{t}}=1$, otherwise, $\mathrm{M}_{\mathrm{k}, \mathrm{t}}=0$. Then normalize the pattern weight.

$\mathrm{w}_{\mathrm{k}, \mathrm{t}}=(1-\alpha) * \mathrm{w}_{\mathrm{k}, \mathrm{t}-1}+\alpha * \mathrm{M}_{\mathrm{k}, \mathrm{t}}(5)$

4)For no matched mode, mean $\mu$ and standard deviation $\sigma$ remain the same, the matching mode parameters are pressed type: 


$$
\left\{\begin{array}{c}
\rho=\alpha * \eta\left(X_{\mathrm{t}} \mid \mu_{\mathrm{k}}, \sigma_{\mathrm{k}}\right) \\
\mu_{\mathrm{t}}=(1-\rho) * \mu_{\mathrm{t}-1}+\rho * \mathrm{X}_{\mathrm{t}}
\end{array}\right.
$$

A comparison of the foreground of Gauss's background and non-Gauss background is made by using MATLAB. We found that the effect using the Gauss background modeling is better, as shown in figure 2. It is not difficult to see that the use of Gauss background modeling can be very good to eliminate the noise caused by the tree and shadow.

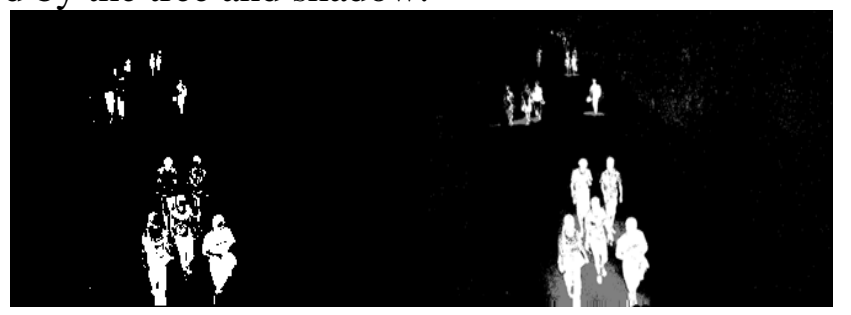

(a) Gauss background modeling (b) General modeling

Fig.2 Comparison between Gauss's background and general background modeling

Canny edge detection operator is a kind of multi-level edge detection algorithm developed by F. Canny John in 1986 [3]. Canny is to find an optimal edge detection algorithm, which mainly includes the following aspects:

1) Must be able to mark the edges of objects in the target image as much as possible.

2) The edges of the logo and the actual edges of the object should be as close as possible.

3) The minimal response edge is identified only once, and those with noise may not be identified as the edge.

Canny gradient detection mainly consists of the following steps: using a Gaussian filter to smooth the image; use first order partial derivatives of the finite difference points to calculate gradient magnitude and direction; the amplitude, non-maxima suppression; using double threshold edge detection algorithm and connected [4].

The functions used by Gauss smoothing are as follows:

$$
\mathrm{h}(\mathrm{x}, \mathrm{y}, \sigma)=\frac{1}{2 \pi \sigma^{2}} \mathrm{e}^{-\frac{\mathrm{x}^{2}+\mathrm{y}^{2}}{2 \sigma^{2}}}(7)
$$

So that $g(x, y)$ is a smooth image, using $h(x, y, \sigma)$ to $f(x, y)$ smooth, the process can be expressed as:

$$
\mathrm{g}(\mathrm{x}, \mathrm{y})=\mathrm{h}(\mathrm{x}, \mathrm{y}, \sigma) * \mathrm{f}(\mathrm{x}, \mathrm{y})(8)
$$

The gradient of $\mathrm{G}(\mathrm{x}, \mathrm{y})$ can be used to calculate the partial derivative of $\mathrm{Y}$ and $\mathrm{X}$ by 2x2:

$$
\left\{\begin{array}{c}
\mathrm{f}_{\mathrm{x}}(\mathrm{x}, \mathrm{y}) \approx \mathrm{G}_{\mathrm{x}}=[\mathrm{f}(\mathrm{x}+1, \mathrm{y})-\mathrm{f}(\mathrm{x}, \mathrm{y}) \\
+\mathrm{f}(\mathrm{x}+1, \mathrm{y}+1)-\mathrm{f}(\mathrm{x}, \mathrm{y}+1)] \\
\mathrm{f}_{\mathrm{y}}(\mathrm{x}, \mathrm{y}) \approx \mathrm{G}_{\mathrm{y}}=[\mathrm{f}(\mathrm{x}, \mathrm{y}+1)-\mathrm{f}(\mathrm{x}, \mathrm{y}) \\
+\mathrm{f}(\mathrm{x}+1, \mathrm{y}+1)-\mathrm{f}(\mathrm{x}+1, \mathrm{y})]
\end{array}\right.
$$

Amplitude and argument:

$$
\left\{\begin{array}{c}
M[x, y]=\sqrt{G_{x}(x, y)^{2}+G_{y}(x, y)^{2}} \\
\left.\theta[x, y]=\tan ^{-1}\left(G_{x}(x, y)\right) / G_{y}(x, y)\right)
\end{array}\right.
$$

Where $M[x, y]$ is the edge strength, the direction of the edge of $\theta[x, y]$ is $M[x, y]$, and the $\theta[x, y]$ is the direction of the edge.

Hough transform by voting algorithm can detect the shape of objects by voting algorithm ${ }^{[5]}$. By calculating the local maximum the process meets the set of the shape. The classical Hough transform is linear detection, and then it is extended to identify the circle and ellipse ${ }^{[6]}$. Hough transform maps a shape of curve or straight line to another coordinate space as a point and the forms a peak, so the shape detection problem is transformed into the statistics of peak ${ }^{[7]}$.

A straight line in the rectangular coordinate system is $\mathrm{y}=\mathrm{kx}+\mathrm{b}$, and by Hough transform it is expressed as $(\mathrm{k}, \mathrm{b})$. The point $\left(\mathrm{x}_{1}, \mathrm{y}_{1}\right)$ is a straight line $\mathrm{y}_{1}=\mathrm{x}_{1} * \mathrm{k}+\mathrm{b}$ in the Cartesian coordinate system, and $(\mathrm{k}, \mathrm{b})$ is an arbitrary point on the line. First, carry out the edge detection. Then transform 
each nonzero pixel into a straight line in the parameter coordinates. So the principle can be used to detect the line.

\section{Algorithm design}

The algorithm's design flow of detection of moving stick like objects is shown in Fig.3.

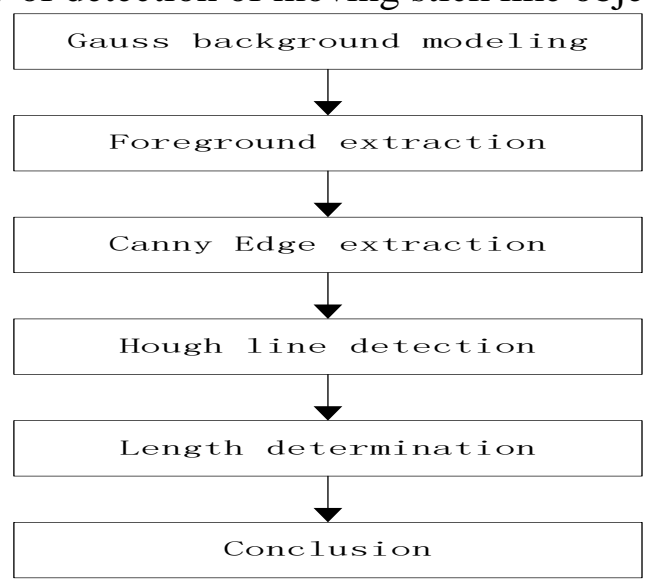

Fig.3 Design flow of detection

The foreground extraction using Gaussian is not perfect, sometimes with small noise, especially when there is tree shaking or light micro change happening in the scene. Grainy noise exclusion is done by using open operation. Nonstick like objects such as the edge of the roof would be detected straight when the light mutation. At that time, we can set a length limit to remove very long lines. Detection stick like objects in that way is more accurate.

\section{Simulation and results}

We carry on the simulation to the above algorithm, through the analysis of a video. The results of the tests are as follows:

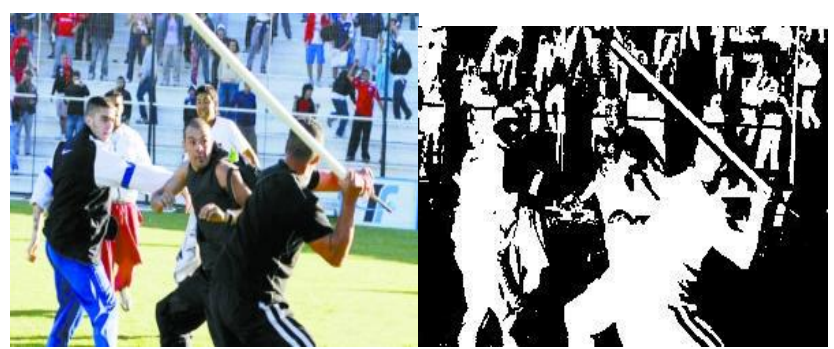

(a)A frame in the source video(b) Foreground image

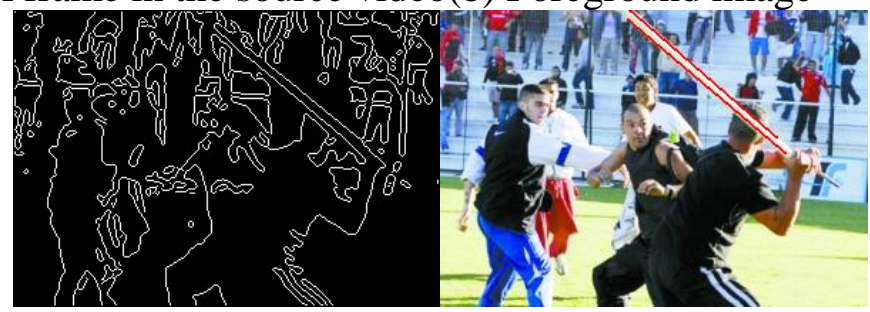

(c) Canny image (d) Result

Fig. 4 results of test

The analysis of the environment of the park with stable light and not too many pedestrians is carried out. Waving the stick 200 times, test it and the statistics is shown in Table 1:

Table 1 Statistical test results of the movement of the stick Waving 100 times(1m stick)

Waving 100 times(0.5m stick)

$\begin{array}{ccc}\text { Right detection } & 89 & 74 \\ \text { Wrong detection } & 11 & 26\end{array}$


The statistics of the mistakes are shown in Table 2:

Table 2 the statistics of the mistakes

\begin{tabular}{cccc}
\hline Waving 100 times(1m stick) & \multicolumn{2}{c}{ Waving 100 times(0.5m stick) } \\
\hline Type & Times & Type & Times \\
Arm & 5 & Arm & 11 \\
Building & 3 & Building & 9 \\
Car & 3 & Car & 6 \\
Total & 11 & Total & 26 \\
\hline
\end{tabular}

\section{Conclusion}

In this paper, the algorithm is tested by means of motion detection and the feasibility of the algorithm is verified by simulation. But there are also some inadequacies, the edge of some cars in the light of the sun will also present like stick; the arm or leg would be detected as stick if it is in the right angle. These are questions to be studied and solved. In addition, it is a good choice to introduce the method of machine learning to find the effective characteristics of the stick like objects, which is the next step of the research.

\section{References}

[1]. Chun-Yan F U, Ping L I, Wen Y M, et al. Gait Silhouette Extraction Algorithm Using Gauss Model[J]. Chinese Journal of Sensors \& Actuators, 2008.

[2]. Zhu J, Liu G D, Liu C L, et al. Oil reserves and production prediction based on multi-cyclic Gauss model[J]. Journal of China University of Petroleum, 2009.

[3]. J. A computational approach to edge detection[J]. IEEE Transactions on Pattern Analysis \& Machine Intelligence, 1986, 8(6):679-698.

[4]. D Demigny, F G Lorca, L Kessal. Evaluation of edge detectors performances with a discrete expression of Canny's criteria[C]// Image Processing, 1995. Proceedings, International Conference on. IEEE, 1995:169 - 172.

[5]. Worthington P L. Enhanced Canny edge detection using curvature consistency[A].Los Alamitos,CA,USA:IEEE,Computer SOC Press,2002.596-599.

[6]. Illingworth J, Kittler J. A survey of the hough transform[J]. Computer Vision Graphics \& Image Processing, 1988, 44(88):87-116.

[7]. Kiryati N, Y. Eldar, A.M. Bruckstein. A probabilistic Hough transform.[J]. Pattern Recognition, 1991, 24(91):303-316. 